Commun. Korean Math. Soc. 28 (2013), No. 2, pp. 303-317

http://dx.doi.org/10.4134/CKMS.2013.28.2.303

\title{
SOME PROPERTIES OF GENERALIZED HYPERGEOMETRIC FUNCTION
}

\author{
Snehal B. Rao, Amit D. Patel, Jyotindra C. Prajapati, \\ AND AJAY K. SHUKLA
}

\begin{abstract}
In present paper, we obtain functions $R_{t}(c, \nu, a, b)$ and $R_{t}(c$, $-\mu, a, b)$ by using generalized hypergeometric function. A recurrence relation, integral representation of the generalized hypergeometric function ${ }_{2} R_{1}(a, b ; c ; \tau ; z)$ and some special cases have also been discussed.
\end{abstract}

\section{Introduction and preliminaries}

The special functions play very important role, particularly the hypergeometric function in solving numerous problems of mathematical physics, engineering and applied mathematics, is well-known ([4], [6], [9], [15]). This fact has inspired many mathematicians for investigations of several generalizations of hypergeometric function ([5], [11], [16], [17], [18], [19], [20]).

The Gauss hypergeometric function is defined [12] as,

$$
{ }_{2} F_{1}(a, b ; c ; z)=\sum_{k=0}^{\infty} \frac{(a)_{k}(b)_{k}}{(c)_{k} k !} z^{k} \quad(|z|<1, c \neq 0,-1,-2, \ldots)
$$

and the generalized hypergeometric function, in a classical sense, has been defined [3] by

$$
\begin{aligned}
{ }_{p} F_{q}\left[\begin{array}{c}
a_{1}, \ldots, a_{p} ; z \\
b_{1}, \ldots, b_{q}
\end{array}\right] & ={ }_{p} F_{q}\left[a_{1}, \ldots, a_{p} ; b_{1}, \ldots, b_{q} ; z\right] \\
& =\sum_{k=0}^{\infty} \frac{\left(a_{1}\right)_{k} \ldots\left(a_{p}\right)_{k}}{\left(b_{1}\right)_{k} \ldots\left(b_{q}\right)_{k}} \frac{z^{k}}{k !} \quad(p=q+1,|z|<1)
\end{aligned}
$$

and no denominator parameter equals zero or negative integer.

Received May 9, 2012; Revised December 26, 2012.

2010 Mathematics Subject Classification. Primary 33C20, 33E20, 26A33.

Key words and phrases. generalized hypergeometric function, recurrence relation, integral representation, fractional integral and differential operators. 
E. Wright [21] has further extended the generalization of the hypergeometric series in the following form:

$$
{ }_{p} \Psi_{q}(z)=\sum_{n=0}^{\infty} \frac{\Gamma\left(\alpha_{1}+\beta_{1} n\right) \ldots \Gamma\left(\alpha_{p}+\beta_{p} n\right)}{\Gamma\left(\rho_{1}+\mu_{1} n\right) \ldots \Gamma\left(\rho_{q}+\mu_{q} n\right)} \frac{z^{n}}{n !},
$$

where $\beta_{r}$ and $\mu_{t}$ are real positive numbers such that $1+\sum_{t=1}^{q} \mu_{t}-\sum_{r=1}^{p} \beta_{r}>0$.

When $\beta_{r}$ and $\mu_{t}$ are equal to 1, Equation (3) differs from the generalized hypergeometric function ${ }_{p} F_{q}$ by a constant multiplier only. This generalized form of the hypergeometric function has been investigated by M. Dotsenko [2], V. Malovichko [8] and others. One of the interesting special case considered in [2] has the following form:

(4) ${ }_{2} R_{1}^{\omega, \mu}(z)={ }_{2} R_{1}(a, b ; c ; \omega, \mu ; z)=\frac{\Gamma(c)}{\Gamma(a) \Gamma(b)} \sum_{n=0}^{\infty} \frac{\Gamma(a+n) \Gamma\left(b+\frac{\omega}{\mu} n\right)}{\Gamma\left(c+\frac{\omega}{\mu} n\right)} \frac{z^{n}}{n !} ;$

$$
(\Re(a)>0, \Re(b)>0, \Re(c)>0) .
$$

Here $\omega, \mu$ both either positive or negative simultaneously, $|z|<1$.

The function ${ }_{2} R_{1}^{\omega, \mu}(z)$ is not symmetric with respect to the parameters $a$ and $b$. By letting $\frac{\omega}{\mu}=\tau>0$ in Equation (4), Virchenko et al. [18] defined the generalized hypergeometric function in a different sense as:

(5) ${ }_{2} R_{1}^{\tau}(z)={ }_{2} R_{1}(a, b ; c ; \tau ; z)=\frac{\Gamma(c)}{\Gamma(b)} \sum_{k=0}^{\infty} \frac{(a){ }_{k} \Gamma(b+\tau k)}{\Gamma(c+\tau k) k !} z^{k} ; \tau>0,|z|<1$.

If $\tau=1$, then (5) reduces to a Gauss's hypergeometric function ${ }_{2} F_{1}(a, b ; c ; z)$. For $\tau>0$, on $|z|=1$, the function ${ }_{2} R_{1}(a, b ; c ; \tau ; z)$ is defined provided $\Re(c-a-b)>0$, as discussed in the proposition of Appendix A.

Rao et al. [13], [14] studied various properties of generalized hypergeometric function in the light of fractional calculus.

The Riemann-Liouville fractional integral of order $\nu$ is defined as [10]:

For $\Re(\nu)>0$,

$$
I^{\nu} f(t)=\frac{1}{\Gamma(\nu)} \int_{0}^{t}(t-\xi)^{\nu-1} f(\xi) d \xi
$$

and the fractional differential operator of order $\mu$ defined as [10]:

$$
D^{\mu} f(t)=D^{n}\left\{I^{n-\mu} f(t)\right\},
$$

where $\Re(\mu)>0$, and $n$ is the smallest integer with the property that $n>\Re \mu$.

The Laplace transform of the function $f(z)$ is defined as [1]:

$$
L\{f(z)\}=\int_{0}^{\infty} e^{-s z} f(z) d z .
$$




\section{Fractional operators and the generalized hypergeometric function ${ }_{2} R_{1}(a, b ; c ; \tau ; z)$}

Consider the function $f(t)=\frac{1}{\Gamma(b)} \sum_{k=0}^{\infty} \frac{(a)_{k} \Gamma(b+k)(c t)^{k}}{(k !)^{2}}={ }_{2} F_{1}(a, b ; 1 ; c t)$, where $a, b \in \mathbb{C}(\Re(a)>0, \Re(b)>0)$ and $c$ is arbitrary constant such that $|c t|<1$.

On applying the fractional integral operator (6) of order $\nu$ on $f(t)$, we give

$$
\begin{aligned}
I^{\nu} f(t) & =\frac{1}{\Gamma(\nu)} \int_{0}^{t}\left[(t-\xi)^{\nu-1} f(\xi)\right] d \xi \\
& =\frac{1}{\Gamma(\nu)} \int_{0}^{t}\left[(t-\xi)^{\nu-1} \frac{1}{\Gamma(b)} \sum_{k=0}^{\infty} \frac{(a)_{k} \Gamma(b+k)(c \xi)^{k}}{(k !)^{2}}\right] d \xi \\
& =\frac{t^{\nu}}{\Gamma(\nu+1)}\left\{\frac{\Gamma(\nu+1)}{\Gamma(b)} \sum_{k=0}^{\infty} \frac{(a)_{k} \Gamma(b+k)(c t)^{k}}{\Gamma(\nu+1+k) k !}\right\},
\end{aligned}
$$

one can easily write this in following form:

$$
\frac{t^{\nu}}{\Gamma(\nu+1)}{ }_{2} R_{1}(a, b ; \nu+1 ; 1 ; c t)=\frac{t^{\nu}}{\Gamma(\nu+1)}{ }_{2} F_{1}(a, b ; \nu+1 ; c t) .
$$

Here we denote $(9)$ as $R_{t}(c, \nu, a, b)$, i.e.,

$$
\begin{aligned}
R_{t}(c, \nu, a, b) & =\frac{t^{\nu}}{\Gamma(\nu+1)}{ }_{2} R_{1}(a, b ; \nu+1 ; 1 ; c t) \\
& =\frac{t^{\nu}}{\Gamma(\nu+1)}{ }_{2} F_{1}(a, b ; \nu+1 ; c t) .
\end{aligned}
$$

Now, applying the fractional differential operator (7) of order $\mu$ on $f(t)$, we get

$$
\begin{aligned}
D^{\mu} f(t) & =\left(\frac{d}{d t}\right)^{n}\left[I^{n-\mu} \frac{1}{\Gamma(b)} \sum_{k=0}^{\infty} \frac{(a)_{k} \Gamma(b+k)(c t)^{k}}{(k !)^{2}}\right] \\
& =D^{n}\left[\frac{t^{n-\mu}}{\Gamma(b)}\left\{\sum_{k=0}^{\infty} \frac{(a)_{k} \Gamma(b+k)(c t)^{k}}{\Gamma(1+n-\mu+k) k !}\right\}\right]
\end{aligned}
$$

which yields

$$
\begin{aligned}
D^{\mu} f(t) & =\frac{t^{-\mu}}{\Gamma(1-\mu)}{ }_{2} R_{1}(a, b ; 1-\mu ; 1 ; c t) \\
& =\frac{t^{-\mu}}{\Gamma(1-\mu)}{ }_{2} F_{1}(a, b ; 1-\mu ; c t) .
\end{aligned}
$$


On denoting (11) as

$$
\begin{aligned}
R_{t}(c,-\mu, a, b) & =\frac{t^{-\mu}}{\Gamma(1-\mu)}{ }_{2} R_{1}(a, b ; 1-\mu ; 1 ; c t) \\
& =\frac{t^{-\mu}}{\Gamma(1-\mu)}{ }_{2} F_{1}(a, b ; 1-\mu ; c t) .
\end{aligned}
$$

3. Properties of the functions $R_{t}(c, \nu, a, b)$ and $R_{t}(c,-\mu, a, b)$

Theorem 3.1. If $a, b \in \mathbb{C}(\Re(a)>0, \Re(b)>0)$ and $c$ is arbitrary constant such that $|c t|<1$, then

$$
\begin{gathered}
I^{\lambda} R_{t}(c, \nu, a, b)=R_{t}(c, \lambda+\nu, a, b), \\
D^{\lambda} R_{t}(c, \nu, a, b)=R_{t}(c, \nu-\lambda, a, b) .
\end{gathered}
$$

For $n \in \mathbb{N}$ and $\alpha$ as any constant;

The Laplace transform of $R_{t}(c, \nu,-n, \alpha+n-1)$ is given as

$$
L\left\{R_{t}(c, \nu,-n, \alpha+n-1)\right\}=\frac{1}{s^{\nu+1}} \cdot y_{n}(c ; \alpha,-s),
$$

where $y_{n}(c ; \alpha,-s)$ is the generalized Bessel polynomial [7].

Proof. From (6) and the left-hand side of (13), we get

$$
\begin{aligned}
I^{\lambda} R_{t}(c, \nu, a, b) & =\frac{1}{\Gamma(\lambda)} \int_{0}^{t}(t-\xi)^{\lambda-1} R_{\xi}(c, \nu, a, b) d \xi \\
& =\frac{1}{\Gamma(\lambda)} \int_{0}^{t}(t-\xi)^{\lambda-1}\left(\frac{\xi^{\nu}}{\Gamma(\nu+1)}{ }_{2} R_{1}(a, b ; \nu+1 ; 1 ; c \xi)\right) d \xi \\
& =\frac{1}{\Gamma(\lambda)} \int_{0}^{t}(t-\xi)^{\lambda-1}\left(\frac{\xi^{\nu}}{\Gamma(b)} \sum_{k=0}^{\infty} \frac{(a)_{k} \Gamma(b+k)(c \xi)^{k}}{\Gamma(\nu+1+k) k !}\right) d \xi
\end{aligned}
$$

which gives

$$
\begin{aligned}
& I^{\lambda} R_{t}(c, \nu, a, b) \\
= & \frac{1}{\Gamma(\lambda)} \int_{0}^{1}(1-x)^{\lambda-1} t^{\lambda-1}\left(\frac{(x t)^{\nu}}{\Gamma(b)} \sum_{k=0}^{\infty} \frac{(a)_{k} \Gamma(b+k)(c x t)^{k}}{\Gamma(\nu+1+k) k !}\right) t d x \\
= & \frac{t^{\lambda+\nu}}{\Gamma(\lambda+\nu+1)}{ }_{2} R_{1}(a, b ; \lambda+\nu+1 ; 1 ; c t)=R_{t}(c, \lambda+\nu, a, b) .
\end{aligned}
$$

This is the proof of (13). 
From (7) and left hand side of (14);

$$
\begin{aligned}
D^{\lambda} R_{t}(c, \nu, a, b) & =D^{n}\left[I^{n-\lambda} R_{t}(c, \nu, a, b)\right] \\
& =D^{n}\left[R_{t}(c, n-\lambda+\nu, a, b)\right] \\
& =D^{n}\left[\frac{t^{n-\lambda+\nu}}{\Gamma(n-\lambda+\nu+1)}{ }_{2} R_{1}(a, b ; n-\lambda+\nu+1 ; 1 ; c t)\right] \\
& =\frac{t^{\nu-\lambda}}{\Gamma(\nu-\lambda+1)}{ }_{2} R_{1}(a, b ; \nu-\lambda+1 ; 1 ; c t) \\
& =R_{t}(c, \nu-\lambda, a, b)
\end{aligned}
$$

which is (14).

On setting $n \in \mathbb{N}$, replacing $a$ by $-n$ and $b$ by $\alpha+n-1$ in $R_{t}(c, \nu, a, b)$, where $\alpha$ as any constant and then taking Laplace transform of (10), it yields

$$
\begin{aligned}
& L\left\{R_{t}(c, \nu,-n, \alpha+n-1)\right\} \\
= & L\left\{\frac{t^{\nu}}{\Gamma(\nu+1)}{ }_{2} R_{1}(-n, \alpha+n-1 ; \nu+1 ; 1 ; c t)\right\} \\
= & \int_{0}^{\infty} e^{-s t}\left\{\frac{t^{\nu}}{\Gamma(\nu+1)}{ }_{2} R_{1}(-n, \alpha+n-1 ; \nu+1 ; 1 ; c t)\right\} d t \\
= & \frac{1}{s^{\nu+1}} \sum_{k=0}^{n} \frac{(-n)_{k}(\alpha+n-1)_{k}}{k !}\left(\frac{c}{s}\right)^{k} \\
= & \frac{1}{s^{\nu+1}}{ }_{2} F_{0}\left(-n, \alpha+n-1 ;-;-\frac{c}{-s}\right) \\
= & \frac{1}{s^{\nu+1}} \cdot y_{n}(c ; \alpha,-s) .
\end{aligned}
$$

Thus, the Laplace transform of $R_{t}(c, \nu,-n, \alpha+n-1)$ is

$$
L\left\{R_{t}(c, \nu,-n, \alpha+n-1)\right\}=\frac{1}{s^{\nu+1}} \cdot y_{n}(c ; \alpha, s),
$$

where $y_{n}(c ; \alpha,-s)$ is the generalized Bessel polynomial [7].

This proves (15).

Theorem 3.2. Let $a, b \in \mathbb{C}(\Re(a)>0, \Re(b)>0)$ and $c$ is arbitrary constant such that $|c t|<1, \Re(\mu)<1$. Then

$$
\begin{aligned}
I^{\lambda} R_{t}(c,-\mu, a, b) & =R_{t}(c, \lambda-\mu, a, b), \\
D^{\lambda} R_{t}(c,-\mu, a, b) & =R_{t}(c,-\lambda-\mu, a, b) .
\end{aligned}
$$

For $n \in \mathbb{N}$ and $\alpha$ as any constant;

The Laplace transform of $R_{t}(c,-\mu,-n, \alpha+n-1)$ is given as

$$
L\left\{R_{t}(c,-\mu,-n, \alpha+n-1)\right\}=\frac{1}{s^{1-\mu}} \cdot y_{n}(c ; \alpha,-s),
$$

where $y_{n}(c ; \alpha,-s)$ is the generalized Bessel polynomial [7]. 
Proof. From (6) and left hand side of (16), we get

$$
\begin{aligned}
I^{\lambda} R_{t}(c,-\mu, a, b) & =\frac{1}{\Gamma(\lambda)} \int_{0}^{t}(t-\xi)^{\lambda-1} R_{\xi}(c,-\mu, a, b) d \xi \\
& =\frac{1}{\Gamma(\lambda)} \int_{0}^{t}(t-\xi)^{\lambda-1}\left(\frac{\xi^{-\mu}}{\Gamma(b)} \sum_{k=0}^{\infty} \frac{(a)_{k} \Gamma(b+k)(c \xi)^{k}}{\Gamma(1-\mu+k) k !}\right) d \xi
\end{aligned}
$$

which, upon substituting $\xi=x t$, yields

$$
\begin{aligned}
& I^{\lambda} R_{t}(c,-\mu, a, b) \\
= & \frac{1}{\Gamma(\lambda)} \int_{0}^{1}(1-x)^{\lambda-1} t^{\lambda-1}\left(\frac{(x t)^{-\mu}}{\Gamma(b)} \sum_{k=0}^{\infty} \frac{(a)_{k} \Gamma(b+k)(c x t)^{k}}{\Gamma(1-\mu+k) k !}\right) t d x \\
= & \frac{t^{\lambda-\mu}}{\Gamma(\lambda-\mu+1)}{ }_{2} R_{1}(a, b ; \lambda-\mu+1 ; 1 ; c t)=R_{t}(c, \lambda-\mu, a, b) .
\end{aligned}
$$

This is the proof of (16).

From (7) and left hand side of (17), we get

$$
\begin{aligned}
& D^{\lambda} R_{t}(c,-\mu, a, b) \\
= & D^{n}\left[I^{n-\lambda} R_{t}(c,-\mu, a, b)\right] \\
= & D^{n}\left[R_{t}(c, n-\lambda-\mu, a, b)\right] \\
= & D^{n}\left[\frac{t^{n-\lambda-\mu}}{\Gamma(n-\lambda-\mu+1)}{ }_{2} R_{1}(a, b ; n-\lambda-\mu+1 ; 1 ; c t)\right] \\
= & \frac{t^{-\mu-\lambda}}{\Gamma(-\mu-\lambda+1)}\left[\frac{\Gamma(-\mu-\lambda+1)}{\Gamma(b)} \sum_{k=0}^{\infty} \frac{(a)_{k} \Gamma(b+k)}{\Gamma(-\lambda-\mu+1+k)} \frac{(c t)^{k}}{k !}\right] \\
= & \frac{t^{-\mu-\lambda}}{\Gamma(-\mu-\lambda+1)}{ }_{2} R_{1}(a, b ;-\mu-\lambda+1 ; 1 ; c t),
\end{aligned}
$$

this can also be written as $R_{t}(c,-\mu-\lambda, a, b)$. This leads to (17).

On setting $n \in \mathbb{N}$, replacing $a$ by $-n$ and $b$ by $\alpha+n-1$ in $R_{t}(c,-\mu, a, b)$, where $\alpha$ as any constant and then taking Laplace transform of (12), it yields

$$
\begin{aligned}
& L\left\{R_{t}(c,-\mu,-n, \alpha+n-1)\right\} \\
= & L\left\{\frac{t^{\nu}}{\Gamma(1-\mu)}{ }_{2} R_{1}(-n, \alpha+n-1 ; 1-\mu ; 1 ; c t)\right\} \\
= & \int_{0}^{\infty} e^{-s t}\left\{\frac{t^{\nu}}{\Gamma(1-\mu)}{ }_{2} R_{1}(-n, \alpha+n-1 ; 1-\mu ; 1 ; c t)\right\} d t \\
= & \frac{1}{s^{-\mu+1}} \sum_{k=0}^{n} \frac{(-n)_{k}(\alpha+n-1)_{k}}{k !}\left(\frac{c}{s}\right)^{k} \\
= & \frac{1}{s^{1-\mu}}{ }_{2} F_{0}\left(-n, \alpha+n-1 ;-;-\frac{c}{-s}\right)=\frac{1}{s^{1-\mu}} \cdot y_{n}(c ; \alpha,-s) .
\end{aligned}
$$


Thus, the Laplace transform of $R_{t}(c,-\mu,-n, \alpha+n-1)$ is given as

$$
L\left\{R_{t}(c,-\mu,-n, \alpha+n-1)\right\}=\frac{1}{s^{1-\mu}} \cdot y_{n}(c ; \alpha,-s),
$$

where $y_{n}(c ; \alpha,-s)$ is the generalized Bessel polynomial [7].

This is the proof of (18).

\section{Recurrence relation for the generalized hypergeometric function}

$$
{ }_{2} R_{1}(a, b ; c ; \tau ; z)
$$

Theorem 4.1. If $\Re(k)>0, \Re(a)>0, \Re(b)>0, \Re(m)>0,|z|<1$, then

$$
\begin{aligned}
& (m+1){ }_{2} R_{1}(a, b ; c+s+1 ; k ; z)-{ }_{2} R_{1}(a, b ; m+2 ; k ; z) \\
= & \left\{\frac{(k)^{2}}{(m+2)}\right\} z^{2}{ }_{2} \ddot{R}_{1}(a, b ; m+3 ; k ; z)+\frac{(k)}{(m+2)}\{(k)+2(m+1)\} z \\
& \times{ }_{2} \dot{R}_{1}(a, b ; m+3 ; k ; z)+(m){ }_{2} R_{1}(a, b ; m+3 ; k ; z),
\end{aligned}
$$

where ${ }_{2} \dot{R}_{1}(a, b ; c ; \tau ; z)=\frac{d}{d z}{ }_{2} R_{1}(a, b ; c ; \tau ; z)$ and ${ }_{2} \ddot{R}_{1}(a, b ; c ; \tau ; z)=\frac{d^{2}}{d z^{2}}{ }_{2} R_{1}(a$, $b ; c ; \tau ; z)$.

Proof. On applying the fundamental relation of the Gamma function $\Gamma(z+1)$ $=z \Gamma(z)$ to $(5)$, we can write

$$
{ }_{2} R_{1}(a, b ; m+1 ; k ; z)=\frac{\Gamma(m+1)}{\Gamma(b)} \sum_{n=0}^{\infty} \frac{(a)_{n} \Gamma(b+k n)}{(m+k n) \Gamma(m+k n)} \frac{z^{n}}{n !}
$$

$$
{ }_{2} R_{1}(a, b ; m+2 ; k ; z)=\frac{\Gamma(m+2)}{\Gamma(b)} \sum_{n=0}^{\infty} \frac{(a)_{n} \Gamma(b+k n)}{(m+1+k n)(m+k n) \Gamma(m+k n)} \frac{z^{n}}{n !} .
$$

On writing equation (21) as:

$$
\begin{aligned}
& { }_{2} R_{1}(a, b ; m+2 ; k ; z) \\
= & \frac{\Gamma(m+2)}{\Gamma(b)} \sum_{n=0}^{\infty}\left\{\frac{1}{(m+k n)}-\frac{1}{(m+1+k n)}\right\} \frac{(a)_{n} \Gamma(b+k n)}{\Gamma(m+k n)} \frac{z^{n}}{n !} \\
= & (m+1){ }_{2} R_{1}(a, b ; m+1 ; k ; z)-\frac{\Gamma(m+2)}{\Gamma(b)} \sum_{n=0}^{\infty} \frac{(a)_{n} \Gamma(b+k n)}{(m+1+k n) \Gamma(m+k n)} \frac{z^{n}}{n !} .
\end{aligned}
$$

For our convenience, we, denote the last summation in (22) by $S$ as:

$$
\begin{aligned}
S & =\frac{\Gamma(m+2)}{\Gamma(b)} \sum_{n=0}^{\infty} \frac{(a)_{n} \Gamma(b+k n)}{(m+1+k n) \Gamma(m+k n)} \frac{z^{n}}{n !} \\
& =(m+1){ }_{2} R_{1}(a, b ; m+1 ; k ; z)-{ }_{2} R_{1}(a, b ; m+2 ; k ; z) .
\end{aligned}
$$


On applying a simple identity $\frac{1}{u}=\frac{1}{u(u+1)}+\frac{1}{u+1},(u=k n+m+1)$ to $(23)$, we obtain

$$
\begin{aligned}
S= & \frac{\Gamma(m+2)}{\Gamma(b)} \sum_{n=0}^{\infty} \frac{(m+k n)(a)_{n} \Gamma(b+k n)}{\Gamma(m+3+k n)} \frac{z^{n}}{n !} \\
& +\frac{\Gamma(m+2)}{\Gamma(b)} \sum_{n=0}^{\infty} \frac{(c+s+k n)(m+1+k n)(a)_{n} \Gamma(b+k n)}{\Gamma(m+3+k n)} \frac{z^{n}}{n !}
\end{aligned}
$$

and

$$
\begin{aligned}
S= & \frac{k}{(m+2)}\left\{\frac{\Gamma(m+3)}{\Gamma(b)} \sum_{n=1}^{\infty} \frac{(a)_{n} \Gamma(b+k n)}{\Gamma(m+3+k n)} \frac{z^{n}}{(n-1) !}\right\} \\
& +\frac{m}{(m+2)}\left\{\frac{\Gamma(m+3)}{\Gamma(b)} \sum_{n=0}^{\infty} \frac{(a)_{n} \Gamma(b+k n)}{\Gamma(m+3+k n)} \frac{z^{n}}{n !}\right\} \\
& +\frac{k^{2}}{(m+2)}\left\{\frac{\Gamma(m+3)}{\Gamma(b)} \sum_{n=1}^{\infty} \frac{n(a)_{n} \Gamma(b+k n)}{\Gamma(m+3+k n)} \frac{z^{n}}{(n-1) !}\right\} \\
& +\frac{\alpha}{(m+2)}\left\{\frac{\Gamma(m+3)}{\Gamma(b)} \sum_{n=1}^{\infty} \frac{(a)_{n} \Gamma(b+k n)}{\Gamma(m+3+k n)} \frac{z^{n}}{(n-1) !}\right\} \\
& +\frac{\beta}{(m+2)}\left\{\frac{\Gamma(m+3)}{\Gamma(b)} \sum_{n=0}^{\infty} \frac{(a)_{n} \Gamma(b+k n)}{\Gamma(m+3+k n)} \frac{z^{n}}{n !}\right\}
\end{aligned}
$$

where $\alpha=k(2 m+1)$ and $\beta=m(m+1)$.

We now express each summation in the right hand side of (24) as follows:

$$
\begin{aligned}
& \frac{d^{2}}{d z^{2}}\left(z^{2}{ }_{2} R_{1}(a, b ; m+3 ; k ; z)\right) \\
= & 2{ }_{2} R_{1}(a, b ; m+3 ; k ; z)+4 z_{2} \dot{R}_{1}(a, b ; m+3 ; k ; z) \\
& +z^{2}{ }_{2} \ddot{R}_{1}(a, b ; m+3 ; k ; z)
\end{aligned}
$$

and

$$
\text { (26) } \begin{aligned}
& \frac{d^{2}}{d z^{2}}\left(z^{2}{ }_{2} R_{1}(a, b ; m+3 ; k ; z)\right) \\
= & \frac{\Gamma(m+3)}{\Gamma(b)} \sum_{n=0}^{\infty} \frac{(n+2)(n+1)(a)_{n} \Gamma(b+k n)}{\Gamma(m+3+k n)} \frac{z^{n}}{n !} \\
= & \frac{\Gamma(m+3)}{\Gamma(b)} \sum_{n=1}^{\infty} \frac{n(a)_{n} \Gamma(b+k n)}{\Gamma(m+3+k n)} \frac{z^{n}}{(n-1) !} \\
& +3 \frac{\Gamma(m+3)}{\Gamma(b)} \sum_{n=1}^{\infty} \frac{(a)_{n} \Gamma(b+k n)}{\Gamma(m+3+k n)} \frac{z^{n}}{(n-1) !}+2_{2} R_{1}(a, b ; m+3 ; k ; z) .
\end{aligned}
$$


(25) and (26) imply

$$
\begin{aligned}
& \frac{\Gamma(m+3)}{\Gamma(b)} \sum_{n=1}^{\infty} \frac{n(a)_{n} \Gamma(b+k n)}{\Gamma(m+3+k n)} \frac{z^{n}}{(n-1) !} \\
= & z^{2}{ }_{2} \ddot{R}_{1}(a, b ; m+3 ; k ; z)+4 z_{2} \dot{R}_{1}(a, b ; m+3 ; k ; z) \\
& -3 \frac{\Gamma(m+3)}{\Gamma(b)} \sum_{n=1}^{\infty} \frac{(a)_{n} \Gamma(b+k n)}{\Gamma(m+3+k n)} \frac{z^{n}}{(n-1) !} .
\end{aligned}
$$

Let

$$
\begin{aligned}
& \frac{d}{d z}\left(z{ }_{2} R_{1}(a, b ; m+3 ; k ; z)\right) \\
= & { }_{2} R_{1}(a, b ; m+3 ; k ; z)+z{ }_{2} \dot{R}_{1}(a, b ; m+3 ; k ; z)
\end{aligned}
$$

and

(29)

$$
\begin{aligned}
& \frac{d}{d z}\left(z_{2} R_{1}(a, b ; m+3 ; k ; z)\right) \\
= & \frac{\Gamma(m+3)}{\Gamma(b)} \sum_{n=0}^{\infty} \frac{(n+1)(a)_{n} \Gamma(b+k n)}{\Gamma(m+3+k n)} \frac{z^{n}}{n !} \\
= & \frac{\Gamma(m+3)}{\Gamma(b)} \sum_{n=1}^{\infty} \frac{(a)_{n} \Gamma(b+k n)}{\Gamma(m+3+k n)} \frac{z^{n}}{(n-1) !}+{ }_{2} R_{1}(a, b ; m+3 ; k ; z) .
\end{aligned}
$$

From (28) and (29), we get

$$
\frac{\Gamma(m+3)}{\Gamma(b)} \sum_{n=1}^{\infty} \frac{(a)_{n} \Gamma(b+k n)}{\Gamma(m+3+k n)} \frac{z^{n}}{(n-1) !}=z_{2} \dot{R}_{1}(a, b ; m+3 ; k ; z) .
$$

Combining (27) and (30), it yields

$$
\begin{aligned}
& \frac{\Gamma(m+3)}{\Gamma(b)} \sum_{n=1}^{\infty} \frac{n(a)_{n} \Gamma(b+k n)}{\Gamma(m+3+k n)} \frac{z^{n}}{(n-1) !} \\
= & z^{2}{ }_{2} \ddot{R}_{1}(a, b ; m+3 ; k ; z)+z_{2} \dot{R}_{1}(a, b ; m+3 ; k ; z) .
\end{aligned}
$$

Now applying (30) and (31) to (24), we get

$$
\begin{aligned}
S= & \left\{\frac{k^{2}}{(m+2)}\right\} z^{2}{ }_{2} \ddot{R}_{1}(a, b ; m+3 ; k ; z) \\
& +\left\{\frac{k^{2}+k+\alpha}{(m+2)}\right\} z_{2} \dot{R}_{1}(a, b ; m+3 ; k ; z) \\
& +\frac{(m+\beta)}{(m+2)}{ }_{2} R_{1}(a, b ; m+3 ; k ; z) .
\end{aligned}
$$


From (22), (23) and (32), we arrive at

$$
\begin{aligned}
& (m+1)_{2} R_{1}(a, b ; m+1 ; k ; z)-{ }_{2} R_{1}(a, b ; m+2 ; k ; z) \\
= & \left\{\frac{k^{2}}{(m+2)}\right\} z^{2}{ }_{2} \ddot{R}_{1}(a, b ; m+3 ; k ; z)+\frac{k}{(m+2)}\{k+2(m+1)\} \\
& \times z_{2} \dot{R}_{1}(a, b ; m+3 ; k ; z)+m_{2} R_{1}(a, b ; m+3 ; k ; z) .
\end{aligned}
$$

\section{Some integral representations of the generalized hypergeometric}

\section{function}

Theorem 5.1. For $k>0, \Re(a)>0, \Re(b)>0, \Re(m)>0, \Re(m-a-b)>0$ we get

(33)

$$
\int_{0}^{1} t^{m}{ }_{2} R_{1}\left(a, b ; m ; k ; t^{k}\right) d t=\frac{{ }_{2} R_{1}(a, b ; m+1 ; k ; 1)}{(m)}-\frac{{ }_{2} R_{1}(a, b ; m+2 ; k ; 1)}{(m)(m+1)} .
$$

Proof. On putting $z=1$ in (23), it yields

$$
\begin{aligned}
& \frac{\Gamma(m)}{\Gamma(b)} \sum_{n=0}^{\infty} \frac{(a)_{n} \Gamma(b+k n)}{(m+1+k n) \Gamma(m+k n) n !} \\
= & \frac{{ }_{2} R_{1}(a, b ; m+1 ; k ; 1)}{m}-\frac{{ }_{2} R_{1}(a, b ; m+2 ; k ; 1)}{(m+1) m} .
\end{aligned}
$$

Let

$$
\text { (35) } \begin{aligned}
\int_{0}^{z} t^{m}{ }_{2} R_{1}\left(a, b ; m ; k ; t^{k}\right) d t & =\int_{0}^{z} t^{m}\left\{\frac{\Gamma(m)}{\Gamma(b)} \sum_{n=0}^{\infty} \frac{(a)_{n} \Gamma(b+k n)}{\Gamma(m+k n)} \frac{t^{k n}}{n !}\right\} d t \\
& =\frac{\Gamma(m)}{\Gamma(b)} \sum_{n=0}^{\infty} \frac{(a)_{n} \Gamma(b+k n) z^{m+1+k n}}{(m+1+k n) \Gamma(m+k n) n !} .
\end{aligned}
$$

Now, comparing (34) and (35) after setting $z=1$ in (35), we have

$$
\int_{0}^{1} t^{m}{ }_{2} R_{1}\left(a, b ; m ; k ; t^{k}\right) d t=\frac{{ }_{2} R_{1}(a, b ; m+1 ; k ; 1)}{m}-\frac{{ }_{2} R_{1}(a, b ; m+2 ; k ; 1)}{(m)(m+1)} .
$$

This is the proof of Theorem 5.1.

Theorem 5.2. If $a, b, c, \tau \in \mathbb{C}$ such that $\Re(a)>0, \Re(b)>0, \Re(c)>0, \Re(c-$ $\tau)>0, k>0$ and $|z|<1$, then

$$
\begin{aligned}
& { }_{2} R_{1}(a, b ; c ; \tau ; z) \\
= & k z^{\tau-c} \int_{0}^{\infty} \exp \left(-\frac{t^{k}}{z^{k}}\right) t^{c-\tau-1}\left(\sum_{n=0}^{\infty} \frac{\Gamma(b+\tau n) \Gamma(c)(a)_{n} t^{n}}{\Gamma(b) \Gamma(c+\tau n) \Gamma\left(\frac{c-\tau+n}{k}\right)}\right) d t .
\end{aligned}
$$


Proof. Consider, $\int_{0}^{\infty} \exp \left(-\frac{t^{k}}{z^{k}}\right) t^{c-\tau-1}\left(\sum_{n=0}^{\infty} \frac{\Gamma(b+\tau n) \Gamma(c)(a)_{n} t^{n}}{\Gamma(b) \Gamma(c+\tau n) \Gamma\left(\frac{c-\tau+n}{k}\right)}\right) d t$ and substituting $\frac{t^{k}}{z^{k}}=u$, we get

$$
\begin{aligned}
& =\int_{0}^{\infty} e^{-u} z^{c-\tau-1} u^{\frac{c-\tau-1}{k}}\left(\sum_{n=0}^{\infty} \frac{\Gamma(b+\tau n) \Gamma(c) z^{n} u^{\frac{n}{k}}(a)_{n}}{\Gamma(b) \Gamma(c+\tau n) n ! \Gamma\left(\frac{c-\tau+n}{k}\right)}\right) \frac{z}{k} u^{\frac{1-k}{k}} d u \\
& =\frac{z^{c-\tau}}{k} \sum_{n=0}^{\infty} \frac{\Gamma(b+\tau n) \Gamma(c) z^{n}(a)_{n}}{\Gamma(b) \Gamma(c+\tau n) n ! \Gamma\left(\frac{c-\tau+n}{k}\right)} \Gamma\left(\frac{c-\tau+n}{k}\right)
\end{aligned}
$$

which, on further simplification, gives

$$
\begin{aligned}
& \int_{0}^{\infty} \exp \left(-\frac{t^{k}}{z^{k}}\right) t^{c-\tau-1}\left(\sum_{n=0}^{\infty} \frac{\Gamma(b+\tau n) \Gamma(c)(a)_{n} t^{n}}{\Gamma(b) \Gamma(c+\tau n) \Gamma\left(\frac{c-\tau+n}{k}\right)}\right) d t \\
= & \frac{z^{c-\tau}}{k}{ }_{2} R_{1}(a, b ; c ; \tau ; z) .
\end{aligned}
$$

This is the proof of Theorem 5.2.

Theorem 5.3. If $a, b, c, \tau \in \mathbb{C}$ such that $\Re(a)>0, \Re(b)>0, \Re(c)>0, \Re(c-$ $\tau)>0$ and $|z|<1$. Then

$$
{ }_{2} R_{1}(a, b ; c ; \tau ; z)=\frac{\Gamma(c)}{\tau \Gamma(\tau) \Gamma(c-\tau)} \int_{0}^{1}\left(1-t \frac{1}{\tau}\right)_{2}^{c-\tau-1} R_{1}(a, b ; \tau ; \tau ; t z) d t .
$$

Proof. Let

$$
\begin{aligned}
& \int_{0}^{1}\left(1-t^{\frac{1}{\tau}}\right)_{2}^{c-\tau-1}{ }_{2} R_{1}(a, b ; \tau ; \tau ; t z) d t \\
= & \int_{0}^{1}\left(1-t \frac{1}{\tau}\right)^{c-\tau-1}\left(\sum_{n=0}^{\infty} \frac{(a)_{n} \Gamma(b+\tau n) \Gamma(\tau)(t z)^{n}}{\Gamma(b) \Gamma(\tau+\tau n) n !}\right) d t .
\end{aligned}
$$

On substituting $t^{\frac{1}{\tau}}=u$, we get

$$
\begin{aligned}
& \int_{0}^{1}(1-t \bar{\tau})_{2}{ }_{2} R_{1}(a, b ; \tau ; \tau ; t z) d t \\
= & \sum_{n=0}^{\infty} \frac{(a)_{n} \Gamma(b+\tau n) \Gamma(\tau)(z)^{n}}{\Gamma(b) \Gamma(\tau+\tau n) n !} \tau \beta(c-\tau, \tau+\tau n) \\
= & \frac{\tau \Gamma(\tau) \Gamma(c-\tau)}{\Gamma(c)}{ }_{2} R_{1}(a, b ; c ; \tau ; z) .
\end{aligned}
$$


This can easily be written as

${ }_{2} R_{1}(a, b ; c ; \tau ; z)=\frac{\Gamma(c)}{\tau \Gamma(\tau) \Gamma(c-\tau)} \int_{0}^{1}\left(1-t^{\frac{1}{\tau}}\right)^{c-\tau-1}{ }_{2} R_{1}(a, b ; \tau ; \tau ; t z) d t$.

This is the proof of Theorem 5.3.

Theorem 5.4. If $a, b, c, \tau \in \mathbb{C}$ such that $\Re(a)>0, \Re(b)>0, \Re(c)>0, \Re(c-$ $\tau)>0$ and $|z|<1$, then

$$
\begin{aligned}
& { }_{2} R_{1}(a, b ; c ; \tau ; z) \\
= & \frac{\Gamma(c)}{\Gamma(\tau) \Gamma(c-\tau)} \int_{0}^{1} t^{\tau-1}(1-t)_{2}^{c-\tau-1} R_{1}\left(a, b ; c-\tau ; \tau ; z(1-t)^{\tau}\right) d t .
\end{aligned}
$$

Proof.

$$
\begin{aligned}
& \int_{0}^{1} t^{\tau-1}(1-t)_{2}^{c-\tau-1} R_{1}\left(a, b ; c-\tau ; \tau ; z(1-t)^{\tau}\right) d t \\
= & \int_{0}^{1} t^{\tau-1}(1-t)^{c-\tau-1}\left(\frac{\Gamma(c-\tau)}{\Gamma(b)} \sum_{n=0}^{\infty} \frac{(a)_{n} \Gamma(b+\tau n)}{\Gamma(c-\tau+\tau n)} \frac{z^{n}(1-t)^{n \tau}}{n !}\right) d t \\
= & \frac{\Gamma(c-\tau)}{\Gamma(b)} \sum_{n=0}^{\infty} \frac{(a)_{n} \Gamma(b+\tau n)}{\Gamma(c-\tau+\tau n)} \frac{z^{n}}{n !}\left(\int_{0}^{1} t^{\tau-1}(1-t)^{c-\tau+\tau n-1}\right) d t \\
= & \frac{\Gamma(\tau) \Gamma(c-\tau)}{\Gamma(c)}\left\{\frac{\Gamma(c)}{\Gamma(b)} \sum_{n=0}^{\infty} \frac{(a)_{n} \Gamma(b+\tau n)}{\Gamma(c+\tau n)} \frac{z^{n}}{n !}\right\} \\
= & \frac{\Gamma(\tau) \Gamma(c-\tau)}{\Gamma(c)}{ }_{2} R_{1}(a, b ; c ; \tau ; z) .
\end{aligned}
$$

This gives

$$
\begin{aligned}
& { }_{2} R_{1}(a, b ; c ; \tau ; z) \\
= & \frac{\Gamma(c)}{\Gamma(\tau) \Gamma(c-\tau)} \int_{0}^{1} t^{\tau-1}(1-t)_{2}^{c-\tau-1} R_{1}\left(a, b ; c-\tau ; \tau ; z(1-t)^{\tau}\right) d t,
\end{aligned}
$$

which proves Theorem 5.4.

\section{Appendix: A}

Proposition. For $\tau>0$, and $\Re(a)>0, \Re(b)>0, \Re(c)>0$; on $|z|=1$, the function ${ }_{2} R_{1}(a, b ; c ; \tau ; z)$ is defined provided $\Re(c-a-b)>0$.

Proof. For $\tau>0$, and $\Re(a)>0, \Re(b)>0, \Re(c)>0$ the function ${ }_{2} R_{1}(a, b ; c ; \tau$; $z)$ is

$$
{ }_{2} R_{1}^{\tau}(z)={ }_{2} R_{1}(a, b ; c ; \tau ; z)=\frac{\Gamma(c)}{\Gamma(b)} \sum_{n=0}^{\infty} \frac{(a)_{n} \Gamma(b+\tau n)}{\Gamma(c+\tau n) n !} z^{n} .
$$

We have to prove its convergence condition for $|z|=1$ by using the comparison test. 
For $|z|=1, \sum_{n=0}^{\infty} u_{n}=\sum_{n=0}^{\infty}\left|\frac{\Gamma(c)}{\Gamma(b)} \frac{(a)_{n} \Gamma(b+\tau n)}{\Gamma(c+\tau n) n !} z^{n}\right|=1+\sum_{n=1}^{\infty}\left|\frac{\Gamma(c)}{\Gamma(b)} \frac{(a)_{n} \Gamma(b+\tau n)}{\Gamma(c+\tau n) n !}\right|$ and for $\Re(c-a-b)>0, \sum_{n=1}^{\infty} v_{n}=\sum_{n=1}^{\infty} \frac{1}{n^{1+\delta}} ;\left(\delta=\frac{1}{2} \Re(c-a-b)>0\right)$,

$$
\begin{aligned}
& \lim _{n \rightarrow \infty}\left(\frac{u_{n}}{v_{n}}\right) \\
= & \lim _{n \rightarrow \infty}\left(\frac{\left|\frac{\Gamma(c)}{\Gamma(b)} \frac{(a)_{n} \Gamma(b+\tau n)}{\Gamma(c+\tau n) n !}\right|}{\frac{1}{n^{1+\delta}}}\right)=\lim _{n \rightarrow \infty}\left|n^{1+\delta} \frac{\Gamma(c)}{\Gamma(b)} \frac{(a)_{n} \Gamma(b+\tau n)}{\Gamma(c+\tau n) n !}\right| \\
= & \lim _{n \rightarrow \infty}\left|\frac{(a)_{n}}{(n-1) ! n^{a}} \frac{\Gamma(b+\tau n)}{\Gamma(b)(n-1) ! n^{b}} \frac{\Gamma(c)(n-1) ! n^{c}}{\Gamma(c+\tau n)} \frac{(n-1) ! n^{1+\delta}}{n ! n^{c-a-b}}\right| \\
= & \lim _{n \rightarrow \infty}\left|\frac{1}{\Gamma(a)} \frac{\Gamma(b+\tau n)}{\Gamma(b)(n-1) ! n^{b}} \frac{\Gamma(c)(n-1) ! n^{c}}{\Gamma(c+\tau n)}\right| \lim _{n \rightarrow \infty}\left|\frac{1}{n^{c-a-b-\delta}}\right| \\
= & \left(\text { Finite Number). } \lim _{n \rightarrow \infty}\left|\frac{1}{n^{c-a-b-\delta}}\right|(\text { Explanation is given below in Section I })\right. \\
= & 0, \text { because } \Re(c-a-b-\delta)=2 \delta-\delta>0 .
\end{aligned}
$$

Therefore $\sum_{n=0}^{\infty} u_{n}=1+\sum_{n=1}^{\infty}\left|\frac{\Gamma(c)}{\Gamma(b)} \frac{(a)_{n} \Gamma(b+\tau n)}{\Gamma(c+\tau n) n !}\right|$ is convergent, since $\sum_{n=1}^{\infty} v_{n}$ is convergent.

Thus the series in (36) is absolutely convergent on $|z|=1$ when $\Re(c-a-b)$ $>0$.

Hence, ${ }_{2} R_{1}^{\tau}(z)={ }_{2} R_{1}(a, b ; c ; \tau ; z)=\frac{\Gamma(c)}{\Gamma(b)} \sum_{n=0}^{\infty} \frac{(a){ }_{n} \Gamma(b+\tau n)}{\Gamma(c+\tau n) n !} z^{k}$ is convergent for $|z|=1$ when $\Re(c-a-b)>0$.

Section I : For $\Re(a)>0, n \in \mathbb{N}, \tau \geqslant 1,0<|\Gamma(a+n)| \leqslant|\Gamma(a+\tau n)|$. Therefore, $0<\left|\frac{1}{\Gamma(a+\tau n)}\right| \leqslant\left|\frac{1}{\Gamma(a+n)}\right|$.

Thus

$$
0<\lim _{n \rightarrow \infty}\left|\frac{(n-1) ! n^{a}}{\Gamma(a+\tau n)}\right| \leqslant \lim _{n \rightarrow \infty}\left|\frac{(n-1) ! n^{a}}{\Gamma(a+n)}\right| \Rightarrow 0<\lim _{n \rightarrow \infty}\left|\frac{(n-1) ! n^{a}}{\Gamma(a+\tau n)}\right| \leqslant 1 .
$$

Also, for $\Re(a)>0, n \in \mathbb{N}, 0<\tau \leqslant 1,0<|\Gamma(a+\tau n)| \leqslant|\Gamma(a+n)|$.

Which implies that

$$
\begin{aligned}
0<\left|\frac{(n-1) ! n^{a}}{\Gamma(a+n)}\right| \leqslant\left|\frac{(n-1) ! n^{a}}{\Gamma(a+\tau n)}\right| & \Rightarrow 0<\lim _{n \rightarrow \infty}\left|\frac{\Gamma(a+\tau n)}{(n-1) ! n^{a}}\right| \leqslant \lim _{n \rightarrow \infty}\left|\frac{\Gamma(a+n)}{(n-1) ! n^{a}}\right| \\
& \Rightarrow 0<\lim _{n \rightarrow \infty}\left|\frac{\Gamma(a+\tau n)}{(n-1) ! n^{a}}\right| \leqslant 1 .
\end{aligned}
$$


From (37) and (38), afterwards taking the limit $n \rightarrow \infty$ as given below,

$$
\begin{aligned}
& \lim _{n \rightarrow \infty}\left|\frac{1}{\Gamma(a)} \frac{\Gamma(b+\tau n)}{\Gamma(b)(n-1) ! n^{b}} \frac{\Gamma(c)(n-1) ! n^{c}}{\Gamma(c+\tau n)}\right| \\
= & \left|\frac{1}{\Gamma(a)}\right|\left|\lim _{n \rightarrow \infty} \frac{\Gamma(b+\tau n)}{\Gamma(b)(n-1) ! n^{b}}\right|\left|\lim _{n \rightarrow \infty} \frac{\Gamma(c)(n-1) ! n^{c}}{\Gamma(c+\tau n)}\right| .
\end{aligned}
$$

This is finite for all $\tau>0$.

\section{References}

[1] L. Debnath and D. Bhatta, Integral Transforms and Their Applications, Chapman and Hall/CRC press, Boca Raton, FL, 2007.

[2] M. Dotsenko, On some applications of Wright's hypergeometric function, C. R. Acad. Bulgare Sci. 44 (1991), no. 6, 13-16.

[3] A. Erdélyi, W. Magnus, F. Oberhettinger, and F. G. Tricomi, Higher Transcendental Functions. Vols. I, II, McGraw-Hill Book Company, Inc., New York-Toronto-London, 1953.

[4] A. A. Kilbas and M. Saigo, H-Transforms, Chapman and Hall/CRC press, Boca Raton, FL, 2004

[5] A. A. Kilbas, M. Saigo, and J. J. Trujillo, On the generalized Wright function, Fract. Calc. Appl. Anal. 5 (2002), no. 4, 437-460.

[6] V. Kiryakova, Generalized Fractional Calculus and Applications, Wiley \& Sons. Inc., New York, 1994.

[7] H. L. Krall and O. Frink, A new class of orthogonal polynomials: The Bessel polynomials, Trans. Amer. Math. Soc. 65 (1949), 100-115.

[8] V. Malovichko, A generalized hypergeometric function, and some integral operators that contain it, Mat. Fiz. Vyp. 19 (1976), 99-103.

[9] A. M. Mathai and R. K. Saxena, Generalized Hypergeometric Functions With Applications in Statistics and Physical Sciences, Springer-Verlag, Berlin, 1973.

[10] K. S. Miller and B. Ross, An Introduction to the Fractional Calculus and Fractional Differential Equations, John Wiley \& Sons, Inc., 1993.

[11] R. K. Raina, On generalized Wright's hypergeometric functions and fractional calculus operators, East Asian Math. J. 21 (2005), no. 2, 191-203.

[12] E. D. Rainville, Special Functions, The Macmillan Company, New York, 1960.

[13] S. B. Rao, J. C. Prajapati, A. D. Patel, and A. K. Shukla, On generalized hypergeometric function and fractional calculus, Communicated for publication.

[14] S. B. Rao, J. C. Prajapati, and A. K. Shukla, Generalized hypergeometric function and its properties, Communicated for publication.

[15] S. G. Samko, A. A. Kilbas, and O. I. Marichev, Fractional Integrals and Derivatives: Theory and Applications, Gordon and Breach Science publishers, Yverdon (Switzerland), 1993

[16] N. Virchenko, On some generalizations of the functions of hypergeometric type, Fract. Calc. Appl. Anal. 2 (1999), no. 3, 233-244.

[17] On the generalized confluent hypergeometric function and its applications, Fract. Calc. Appl. Anal. 9 (2006), no. 2, 101-108.

[18] N. Virchenko, S. L. Kalla, and A. Al-Zamel, Some results on a generalized hypergeometric function, Integral Transform. Spec. Funct. 12 (2001), no. 1, 89-100.

[19] N. Virchenko, O. Lisetska, and S. L. Kalla, On some fractional integral operators involving generalized Gauss hypergeometric functions, Appl. Appl. Math. 5 (2010), no. 10, 1418-1427. 
[20] N. Virchenko and Olena V. Rumiantseva, On the generalized associated Legendre functions, Fract. Calc. Appl. Anal. 11 (2008), no. 2, 175-185.

[21] E. M. Wright, On the coefficient of the power series having exponential singularities, J. London Math. Soc. 8 (1933), 71-79.

SNehal B. RaO

Department of Applied Mathematics

The M.S. University of Baroda

VADODARA-390 001, INDIA

E-mail address: sbr_msub@yahoo.com

Amit D. Patel

Department of Applied Mathematics and Humanities

S.V. National Institute of Technology

SURAT-395 007, INDIA

E-mail address: ptlamit83@gmail.com

Jyotindra C. Prajapati

Department of Mathematics

Charotar Institute of Technology

Changa, Anand-380 421, India

E-mail address: jyotindra18@rediffmail.com

AJAY K. SHUKLA

Department of Applied Mathematics and Humanities

S.V. National Institute of Technology

Surat-395 007, India

E-mail address: ajayshukla2@rediffmail.com 